\title{
Research on Digital Signal Processing for Motor Control
}

\author{
LIU Yue ${ }^{1, a}$ \\ ${ }^{1}$ Tianjin transportation vocational college, Tianjin 300110, China \\ aliuyue@126.com
}

Keywords: Digital Signal Processing; Motor Control;

\begin{abstract}
With the development of science and technology and the formation of a comprehensive multidisciplinary technology. The emergence of new permanent magnetic actuator and electronic operation of synchronous technology in the field of pressure to achieve a hardware basis. In order to meet the high reliability of the vacuum circuit breaker operation, our country is in-depth study, and gradually promote and electronic control systems match the permanent mechanism. In this article, we have the key technology of digital signal processing theory research motor and motor control. Experimental results show the effectiveness of the method, to better reflect the characteristics of the digital control system for digitizing motion brushless DC motor control engineering applications useful to explore and try.
\end{abstract}

\section{Introduction}

Modern design mutual development and motor control, motor manufacturing technology, microelectronics, power electronics technology combine to produce a number of new motors, such as brushless DC motors, inverter-fed AC motor, switched reluctance motor, an ultrasonic motor . The new motor is an electric motor and electronic control part organically combine to form a indivisible whole, form a system, leaving the control circuit, the motor itself cannot run alone. Naturally, people want to control some of these motors can be integrated. Motor Control predetermined control algorithm to convert into a desired mechanical movement, the controlled mechanical motion control system to achieve precise position control, speed control, acceleration control, torque control, the amount of the controlled machine integrated control. Motor Control reflects the combination of motor technology, sensor technology, power electronics technology, microelectronic technology, automatic control technology and computer application technology. Especially the development of microprocessors and signal processors, dedicated motor control chip appears, giving motor motion control system with intelligence, and to achieve integration. China has been in the theory and practice of synchronous technology for a useful attempt, but the application of synchronous technology in the medium voltage areas have yet to see a report [1-2].

A common problem with the sensor does not automotive driveline is its starting performance. The sensor does not use the above algorithm, the measured signal is too small to make an accurate estimate of the position in a static or low-speed motor. The most common solution is to start an open-loop method from a predetermined position of the rotor. Induced magnetic field direction of the permanent magnet rotor aligns corresponding [3]. With a known initial rotor position, an open loop control applications increase the frequency of injection current speed motors from stagnation. However, in particular three-phase winding of three-phase induction motor, resulting stator field is two opposite directions. With the rapid development of integrated circuits, digital motion control system has been widely used software-based microcontrollers and digital signal processors (DSP) [4]. These methods provide flexibility and suitable motor drive applications.

\section{A digital signal processor for motor control}

Cause develop motor control integrated, modern design mutual development and motor control, motor manufacturing technology, microelectronics, power electronics technology combine to produce a number of new motors, such as brushless DC motors, power inverter AC motors, switched reluctance motors, ultrasonic motors [5-6]. The new motor is an electric motor and 
electronic control part organically combine to form a indivisible whole, form a system, leaving the control circuit, the motor itself cannot run alone. Naturally, people want to control some of these motors can be integrated. Motor Control predetermined control algorithm to convert into a desired mechanical movement, the controlled mechanical motion control system to achieve precise position control, speed control, acceleration control, torque control, the amount of the controlled machine integrated control. Motor Control reflects the combination of motor technology, sensor technology, power electronics technology, microelectronic technology, automatic control technology and computer application technology [7]. Especially the development of microprocessors and signal processors, dedicated motor control chip appears, giving motor motion control system with intelligence, and to achieve integration. The basic idea is used to control motor integrated design are: form a closed loop control of the motor, the motor control algorithm controlled mechanical motion and input the desired motion parameters coincide. Figure 1 shows a complete motor control system, comprising seven basic functional unit.

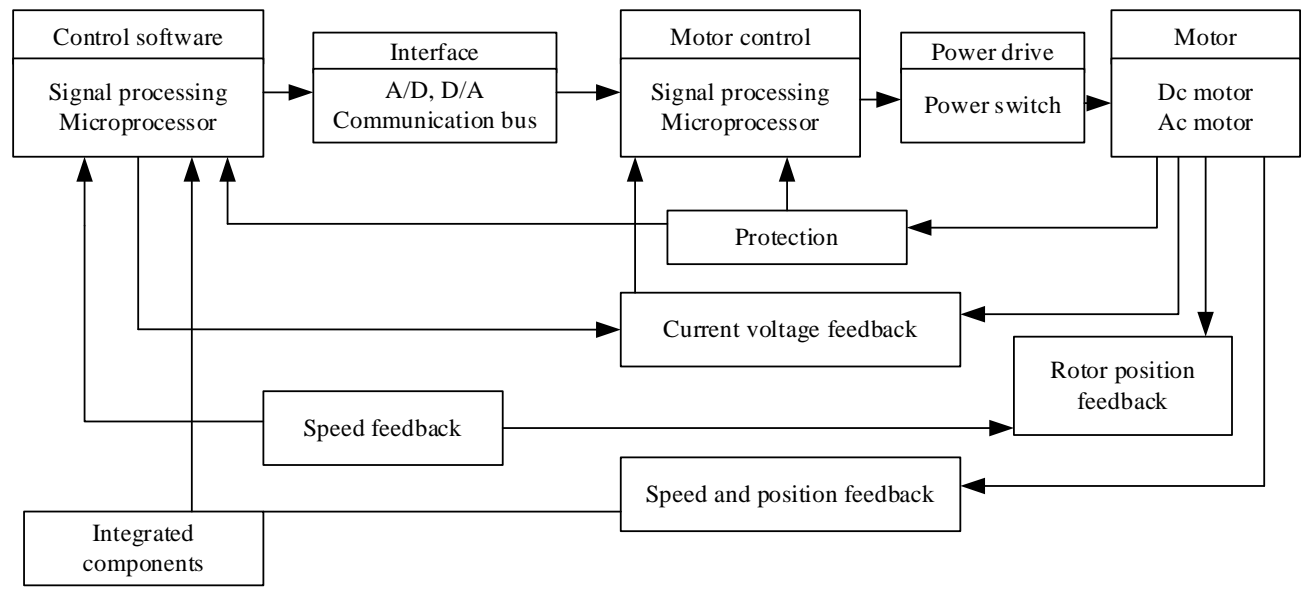

Figure 1. Motor control integrated components

In addition to a microprocessor or digital signal processor cores, the control loop before the channel has three elements: an interface unit, a control unit and a power drive unit. Rotor position unit is designed for brushless DC motors, switched reluctance motor and set the rotor position information channel, providing real-time signal rotor position to generate the correct order of commutation, in synchronous AC servo motor control trapezoidal waveform control aspect, to provide the necessary information to form a composite current feedback signal, and also provide the necessary information for the synthesis of analog tachometer voltage. Speed and position feedback unit is the use of speed and position sensor means producing system outer loop feedback signal. Self-protection unit is a semiconductor device or motor temperature, current, voltage, etc. to detect and monitor, the fault judgment, an alarm signal is transmitted to a digital signal processor or microprocessor cores, or directly off the power output stage. Motor control chip around more cells, to design many superior performance of the circuit.

\section{Our Proposed Methodology}

The Modelling of the Electric Motors. Induction motor and a suitable power converter speed and position feedback, in many complex control applications have become popular. Players' design and compensation system needs an accurate mathematical model of all the components and systems. Excitation coil permanent magnetic actuator by the power electronic control module storage capacitor discharge, completed the closing operation, and a permanent magnet lock, is the perfect combination of electromagnetic systems and permanent magnet systems, and requires force-trip features and vacuum circuit breakers very close. It greatly reduces the transmission links, improve the reliability of response speed, accuracy and running, which is more far-reaching significance is greatly improved controllability, decentralized control of time in milliseconds from the original mechanical system into an electric signal control microseconds progress, from the mechanical energy storage, energy storage mechanical trip progress, the electronic trip signal directly trigger 
action. This study presents a simple modeling approach to meet the characteristics of the three-phase induction motor, and discussed the relationship between static and dynamic driving circuits and the actual movement. Model shown in Fig. 1 . The descriptive equation is shown in the formula 1-3.

$$
\begin{aligned}
& v=L_{s} \frac{d i}{d t}+i R_{s}+v_{e m f} \\
& T_{e}=J_{m} \frac{d w_{r}}{d t}+B_{m} w_{r}+T_{L} \\
& i(t)=\frac{v-v_{e m f}}{R_{s}}\left(1-e^{\left(-R_{s} t / L_{s}\right)}\right)
\end{aligned}
$$

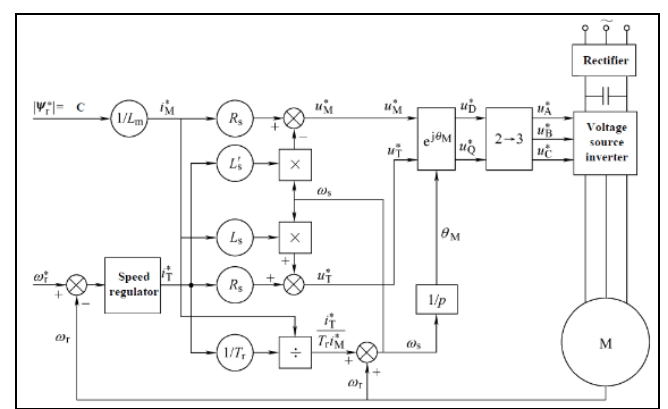

Figure 2. The schematic of the full-bridge inverter for our system

Typical Analog to Digital Conversion. Power converter current and / or voltage feedback, requires that the analog input. Essence synchronization control circuit breaker is open circuit voltage or current phase or near desired. According ungrounded capacitor reactive power compensation in the Star Media Group, for example, the analysis describes the zero reference voltage synchronous switching control method. Sampling data transfer via an interrupt CPU or direct memory access operations by memory sharing mechanism to achieve. Which eliminate the overhead and latency, typically interrupted.

Optimization of Three Phase Induction Motors. Because of the design and structure of the machine, to induce phase induction motor back EMF is highly nonlinear and contains harmonics. From the above analysis, the detection accuracy of the zero reference synchronization control is essential. The main program set up remote control commands (including brake, close the lock and unlock), contact, regular time, the external interrupt flag register, the pressure loss, flow, control voltage limits, such as the circuit breaker contacts impending failure flag register. System self-test and initialization, first determine the system to normal after detecting a control voltage, ambient temperature environmental parameters, such as system voltage, current, and then track the status of the above work, marking the registration fails, such as setting the flag to be found in the corresponding processing subroutine. Common hardware zero compensation voltage comparator method, the impact of higher harmonic and white noise is relatively large. To overcome these drawbacks, digital filtering methods can be used to detect zero. Close-phase minimum target phase delay as shown in Equation 4.

$$
\left\{\begin{array}{l}
t_{d A}=1 / 2 f-\left[t_{c 1 A} \bmod (1 / 2 f)\right]+10.0 \\
t_{d B}=1 / 2 f-\left[t_{c 1 B} \bmod (1 / 2 f)\right]+8.33 \\
t_{d C}=1 / 2 f-\left[t_{c 1 C} \bmod (1 / 2 f)\right]+5.00
\end{array}\right.
$$

\section{Experiment Analysis and Simulation}

Sensor current loop control scheme may not verified by computer simulation model proposed. In this study, the digital PI controller for current regulation, to ensure accurate measurement of the stator current track to the desired value, transient time interval as short as possible. For simplicity, we use the digital design method, which determines the equivalent continuous-time model of the whole system, use it in a stable and continuous time feedback loop controller design, and finally, 
continuous time controller into an equivalent discrete time. Use the advantages of digital design, some of the development controller design method known continuous time models were analyzed. In Figure 3, we show the simulation results.

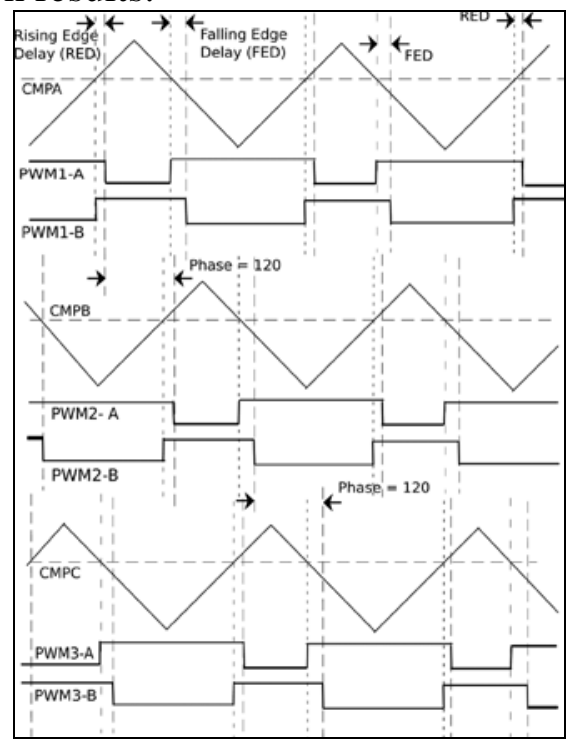

Figure 3. The simulation of our research

For the entire speed range, RMS and peak low when using closed-loop current-mode control. That is, the sensor is not closed-loop motor drive current-mode control is a low cost, high efficiency solution for three-phase induction motors.

\section{Conclusion and Summary}

As a direct result of the use of electrical energy electron linear motion mechanism, a linear motor can directly drive a mechanical load for a linear movement, its outstanding advantages have been making more and more people's attention. Motor control system consists of a displacement detecting device, a controller, isolation circuit, a power amplifier and a host computer, of which the DSP controller is a key part of the motor control system, which is responsible for the displacement of the motor feedback signal processing and outputs a control signal control movement of the motor. DSP controller having particularly suitable for motor control event management module controls the motor so that a simple hardware structure and easy to implement, easy to implement software design. For motor accurately achieve the intended movement, the control software is the key. The entire control program from the main program, general purpose timer period interrupt subroutine, serial port communication routines, subroutines and displacement motor for zero adjustment subroutines. Wherein the shift control is achieved by the PID algorithm. After the completion of the design of the control program, the paper conducted experiments, will draw motor sports during displacement and exercise time into a curve, its control to good effect, proving the control program is feasible.

\section{References}

[1] Jia-yong, C., You-ping, C., Qiong-hua, Z., \& Zu-de, Z. (2002). A review of control technology of switched reluctance motor. Electric Machines and Control, 6, 1, 1-80.

[2] Lu, Z., Sheng, H., Hess, H. L., \& Buck, K. M. (2005). The modeling and simulation of a permanent magnet synchronous motor with direct torque control based on matlab/simulink. Electric Machines and Drives, 2005 IEEE International Conference on, 7 pp. - 1156.

[3] Ping, S., \& Jian-qiu, Y. (2008). Permanent ac servo motor and control system of lockstitch sewing machine. Electric Machines \& Control Application.

[4] Ze-yun, C., Yong, K., He-qing, Z., \& Zhi-xin, X. (2007). Modeling and simulation of 
asynchronous motor vector control system. Electric Machines \& Control Application.

[5] Gonzalez-Llorente, J., Ortiz-Rivera, E. I., \& Diaz, A. (2009). A maximum power point tracker using positive feedforward control based on the dc motor dynamics and pvm mathematical model. Electric Machines and Drives Conference, 2009. IEMDC '09. IEEE International, 259 - 264.

[6] Blum, D. W. (1998). Dynamo-electric machines and control and operating system for the same. US, US6222331 B1.

[7] Zhi-feng, Z., Ren-yuan, T., Jian-guang, Z., \& Bao-dong, B. (2010). Nonsingular terminal sliding mode control of flux and torque for induction motor. Electric Machines and Control 